Supporting Information

\title{
Droplets self-born in dynamic polymer for generating functional coatings
}

Qian Wu ${ }^{\ddagger}, 1$, Li Yang ${ }^{\ddagger}, 1$, Hong Wang ${ }^{1}$, Shihua Dong ${ }^{1}$, Longquan Chen ${ }^{2}$, Juntang Li ${ }^{3}$, Jiaxi Cui ${ }^{*, 1}$.

${ }^{1}$ Institute of Fundamental and Frontier Sciences, University of Electronic Science and Technology of China, Chengdu 610054, P. R. China

${ }^{2}$ School of Physics, University of Electronic Science and Technology of China, Chengdu 610054, P. R. China

${ }^{3}$ Collaborative Innovation Center of Medical Engineering, Luoyang 471031, Henan, China

Corresponding email: Jiaxi.Cui@uestc.edu.cn

\section{Contents}

Supplementary Text

Supplementary Figures S1-12

Supplementary Table

Supplementary References

Supplementary Video 


\section{Supplementary Text}

Swelling ratio $(Q)$ : We measured the swelling ratio by using the gravimetric method. The uPDMS film weighed on an analytical balance with an accuracy of $10^{-4} \mathrm{~g}$ to obtain the mass of the gel, $\mathrm{M}_{\mathrm{uPDMS}}$. Before measurements, the samples gotten from silicone oil bath were cleaned with tissue paper to remove the silicone oil on the surface. The gel was then immediately weighed to obtain the mass of the gel, $\mathrm{M}_{\mathrm{gel}}$.

$$
\mathrm{Q}=\frac{M_{g e l}}{M_{u P D M S}}
$$

Peak Force Quantitative Nanomechanical Mapping (PF-QNM) experiments: PF-QNM measurements were performed by using a Bio Scope Resolve Bio AFM (Bruker, USA) operated in the intermittent mode under ambient conditions at a scan rate of $0.5 \mathrm{~Hz}$. The analysis of the Derjaguin-MuellerToporov (DMT) modulus was performed by the software Nanoscope Analysis. ${ }^{[1]}$

Dye-conjugated silicone oil: PDI-conjugated silicone oil was synthesized according to our previously reported method ${ }^{[2]}$. Briefly, the mixture of perylene-3,4,9,10-tetracarboxylic dianhydride (4 mg, 0.01 mmol) and mono-amino-terminal PDMS (4 g, $1.6 \mathrm{mmol})$ in $5 \mathrm{ml} \mathrm{DMF}$ was stirred at $150{ }^{\circ} \mathrm{C}$ for 7 days under continuous purging with $\mathrm{N}_{2}$ gas. After cooling to room temperature $\left(25^{\circ} \mathrm{C}\right)$, the mixture was washed with water and then filtered through a Teflon membrane (pore size: $0.2 \mu \mathrm{m}$ ). The solvents (water and DMF) were removed under vacuum to produce a red solution, then the red solution $(10 \mu \mathrm{l})$ directly dissolved in silicone oil $(5 \mathrm{cSt}, 50 \mathrm{ml})$ to form dye-conjugated silicone oil and regarded as the swollen liquid.

Droplet Size Analysis: The droplet size and size distribution were determined by optical microscopy, which is calculated by the following formula:

$$
\mathrm{U}=\frac{D_{w}}{D_{n}}=\frac{\sum_{i=1}^{k} n_{i} D_{i}^{4} / \sum_{i=1}^{k} n_{i} D_{i}^{3}}{\sum_{i=1}^{k} n_{i} D_{i} / \sum_{i=1}^{k} n_{i}}
$$


Where $U$ is the polydispersity index, $D_{n}$ is the number-average diameter, $D_{w}$ is the weight-average diameter, $\mathrm{n}$ is the total number of the measured particles, and $\mathrm{D}_{\mathrm{i}}$ is the particle diameters of the determined microspheres..$^{[3]}$ 


\section{Supplementary Figures}

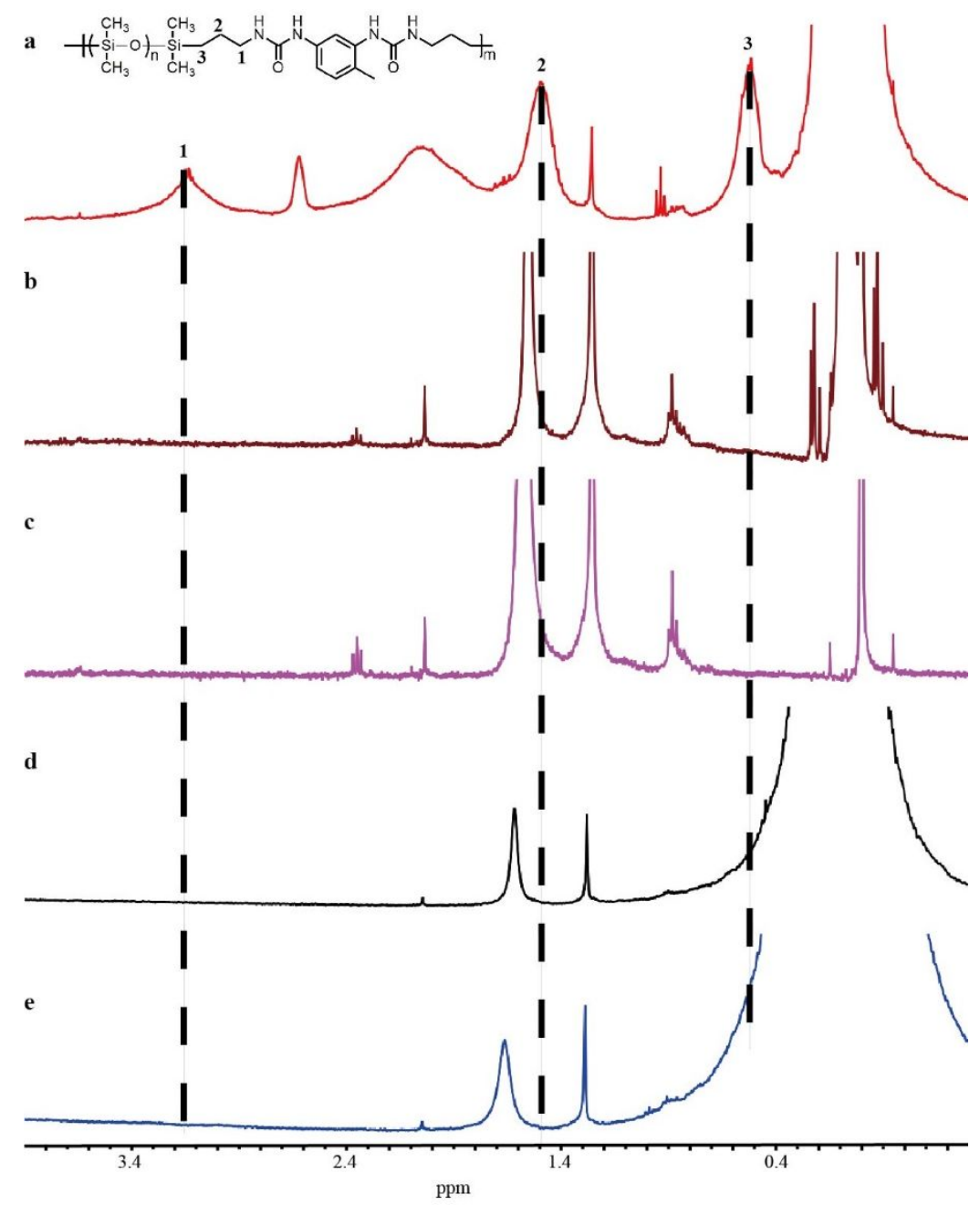

Figure S1. ${ }^{1} \mathrm{H}-\mathrm{NMR}$ spectra of pure uPDMS film (a), the mixture of degreasing cotton and the liquid collected from the droplets (b), degreasing cotton (c), pure silicone oil (d) and residual silicone oil after swelling (e). $\mathrm{CDCl}_{3}$ was used for measurement solvent. The peaks highlighted by numbers 1,2 , and 3 show the typical peak from uPDMS. These peaks are not observed in (b) and (e), which indicated that there is not uPDMS in the droplets and also the residual silicone oil after swelling. In other words, the droplets are pure silicone oil and no uPDMS was dissolved in the silicone oil in our swelling condition. 


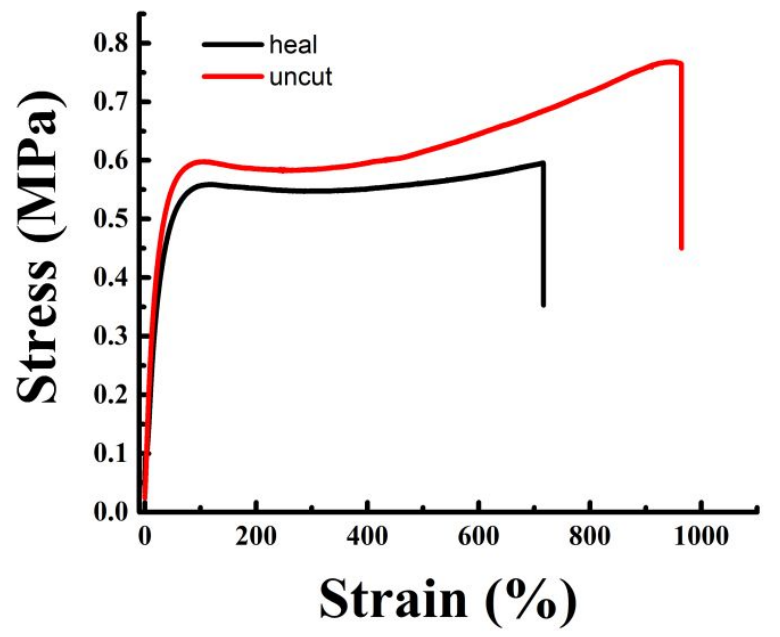

Figure S2. Stress vs strain and self-healing tests of pure uPDMS film. The sample was cut into completely separate pieces using a razor blade and the cut faces were gently brought together and allowed to heal at $50{ }^{\circ} \mathrm{C}$ for $1 \mathrm{~d}$, the self-healing efficiency at 50 ${ }^{\circ} \mathrm{C}$ after one day: $75 \%$. 


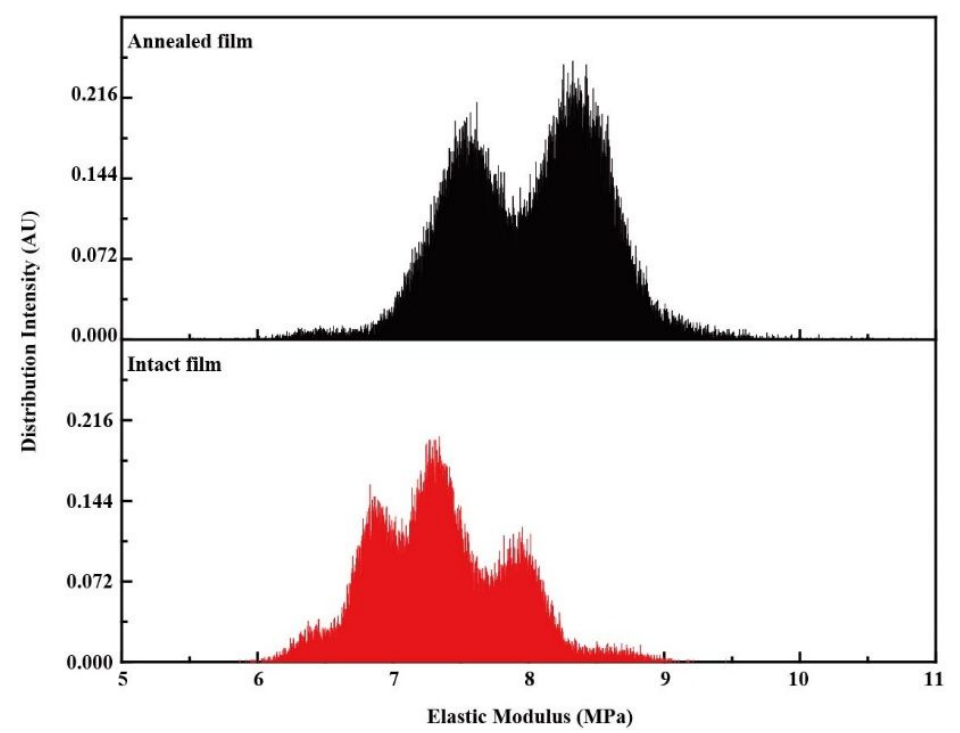

Figure S3. JKR modulus distributions over the entire sample area collected via a Peak Force QNM $\left(500 \times 500 \mathrm{~nm}^{2}\right)$ measurement. The force used for measurement was 300 $\mathrm{pN}$. 


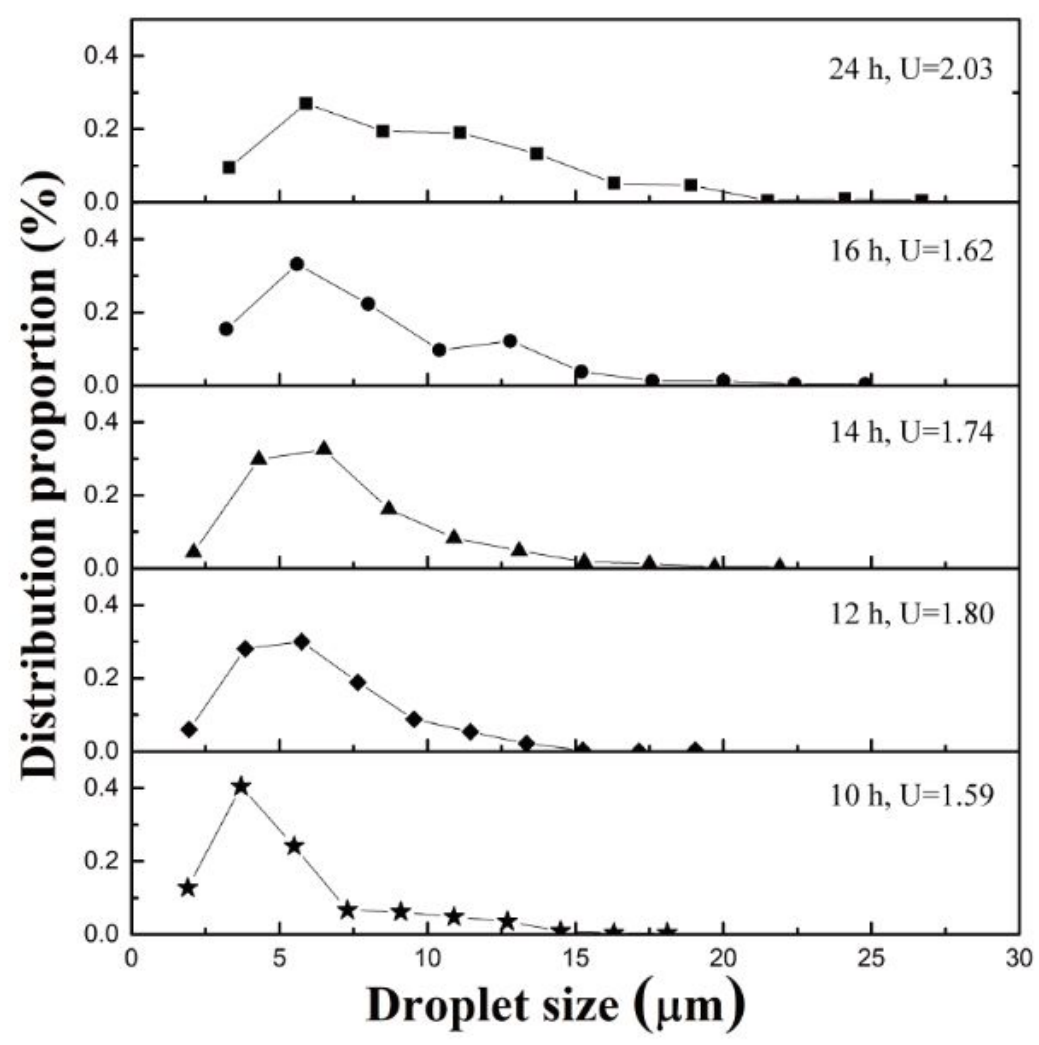

Figure S4. The size distribution of the droplets formed in swollen uPDMS at different immersion times. $\mathrm{U}$ is the polydispersity index. 


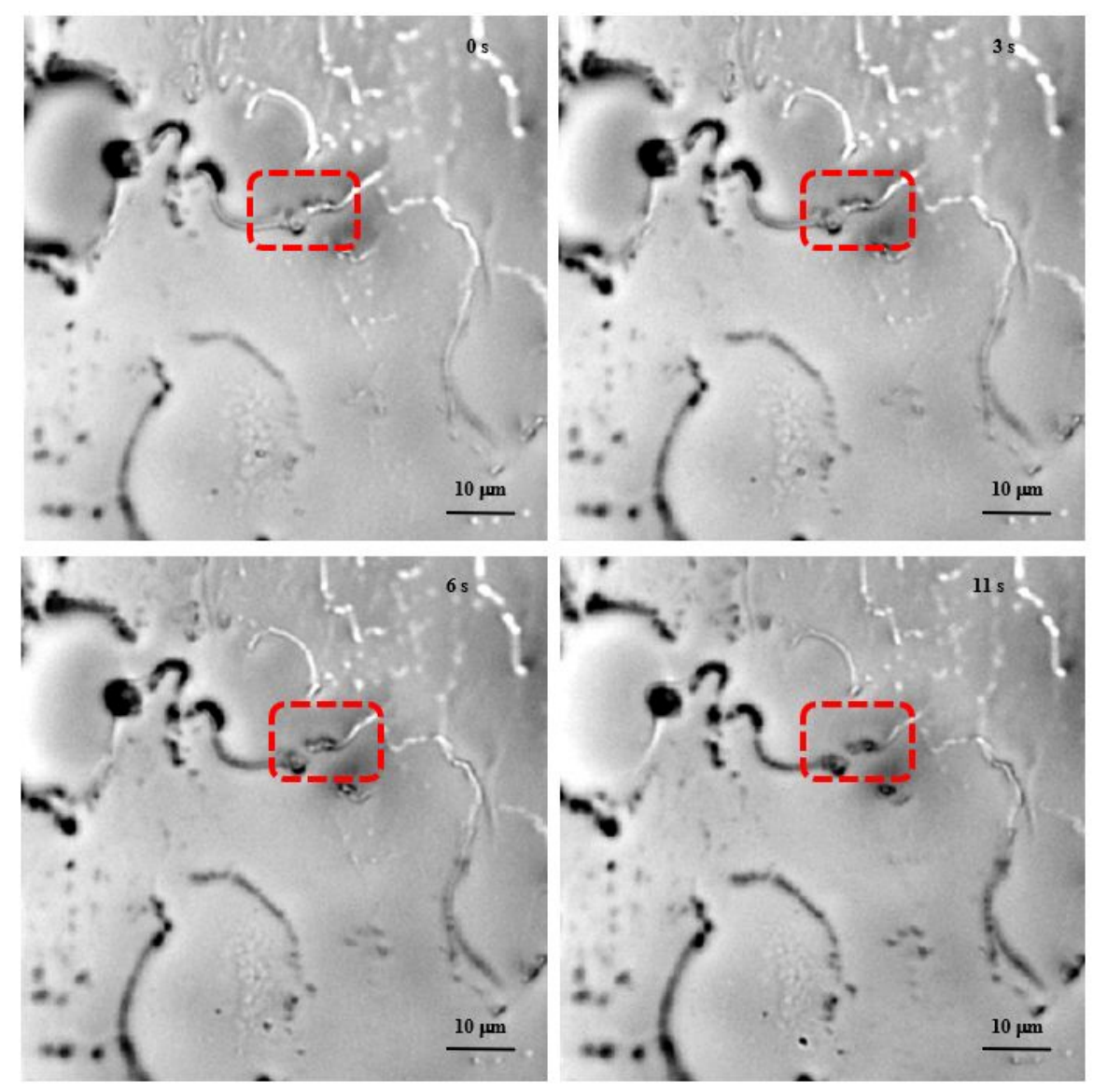

Figure S5. Optical microscopy images of the crossing section surface of the liquid in the uPDMS film. 
a
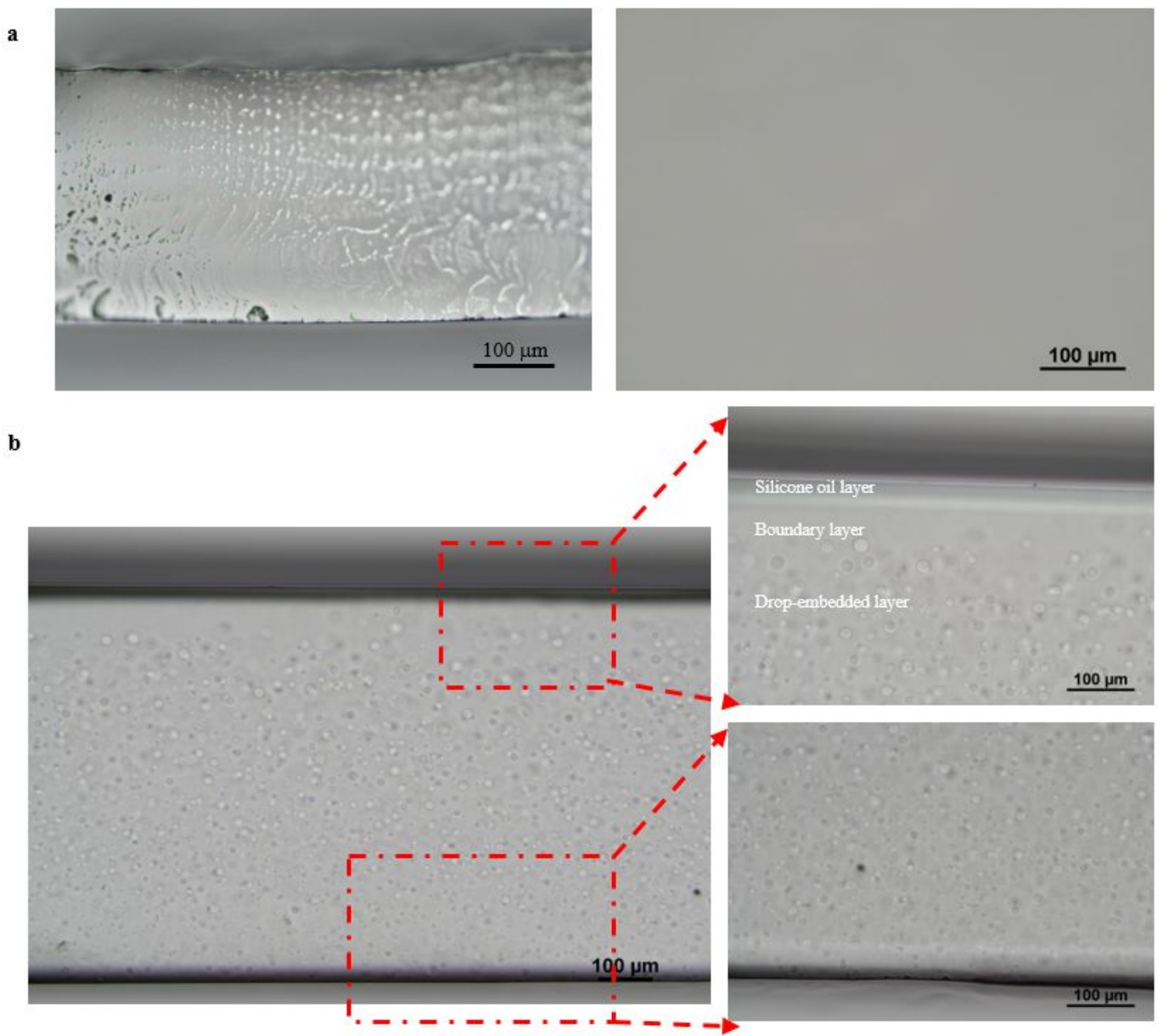

Figure S6. (a) Optical microscopy images of the cross-section and flat of the swollen normal PDMS film. (b) Optical microscopy images of the cross-section of the swollen uPDMS film. 

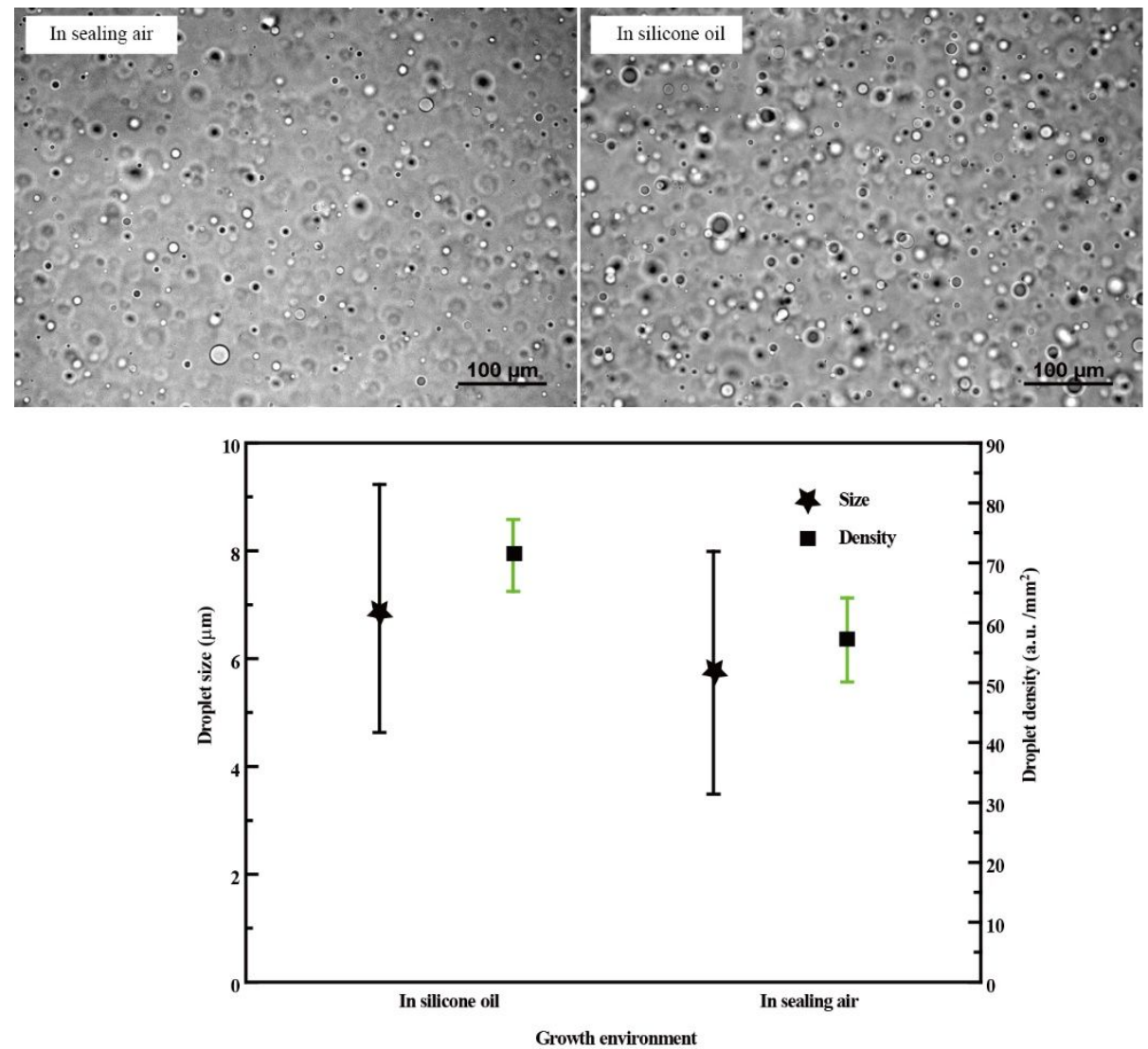

Figure S7. Comparison of swollen uPDMS under different conditions. In sealing air: the uPDMS was immersed in silicone oil $(5 \mathrm{cSt})$ for 8 hours at $80^{\circ} \mathrm{C}$ and then collected from the silicone oil, followed by storage in a sealing petri dish for 10 hours. In silicone oil: the uPDMS was immersed in silicone oil $(5 \mathrm{cSt})$ for 18 hours at $80{ }^{\circ} \mathrm{C}$. 

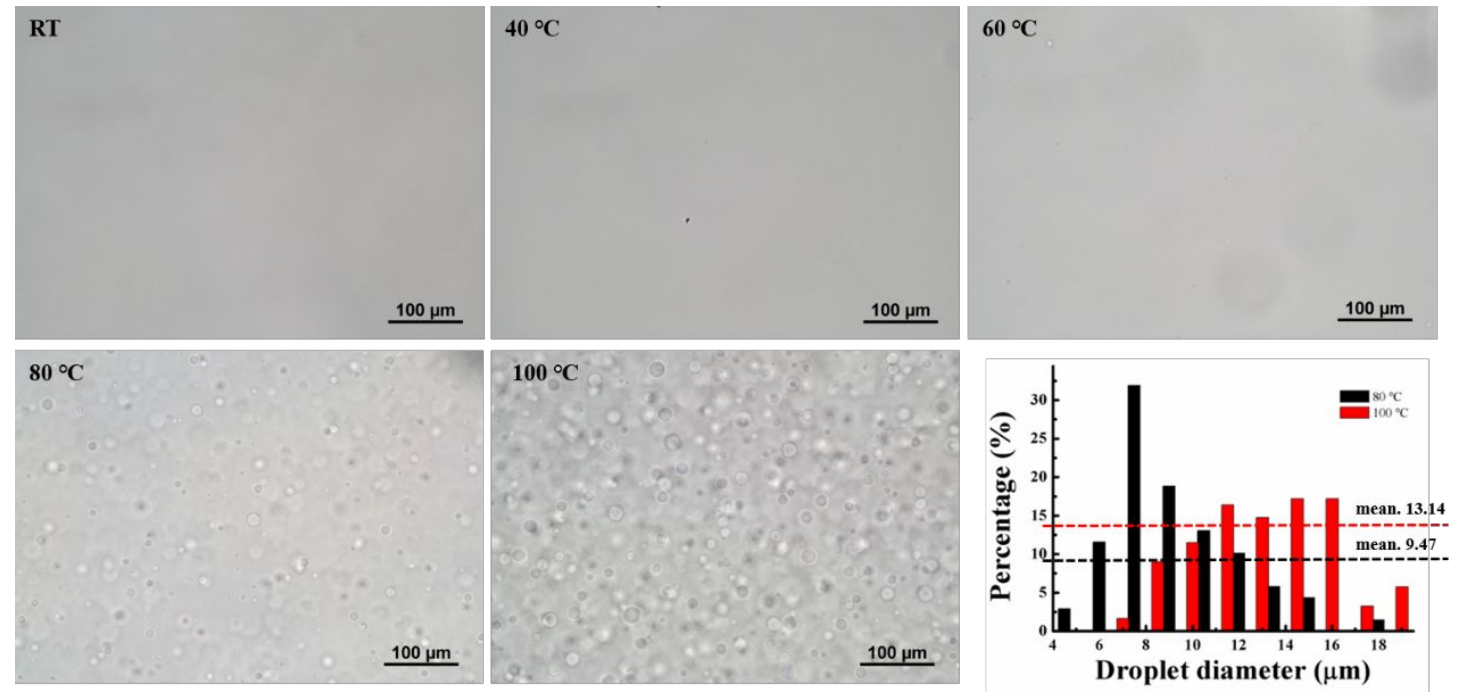

Figure S8. The samples swelled at different temperatures for $24 \mathrm{~h}$. 

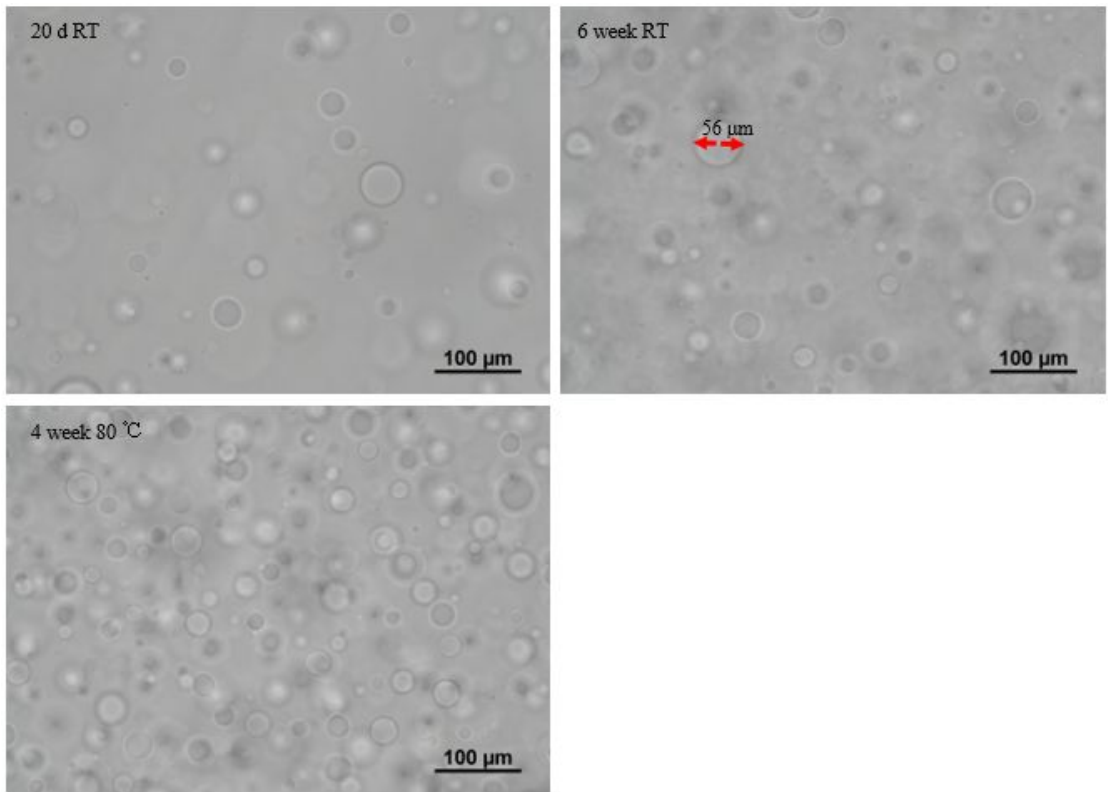

Figure S9. Optical microscopy images of the uPDMS film swelled in silicone oil at different temperatures for long swelling time. 


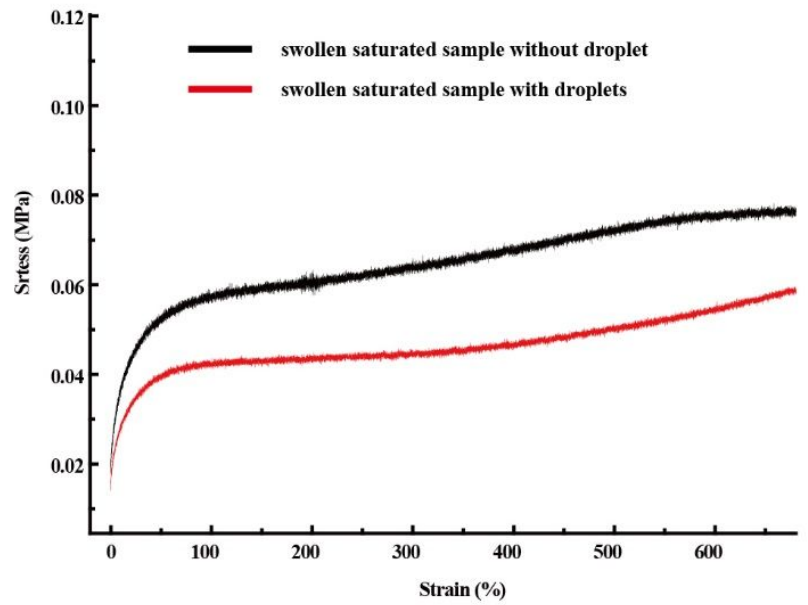

Figure S10. Stress-strain curves of the swollen gel samples with or without droplets.

Both samples were swollen by silicone oil and show the same swelling ration. 


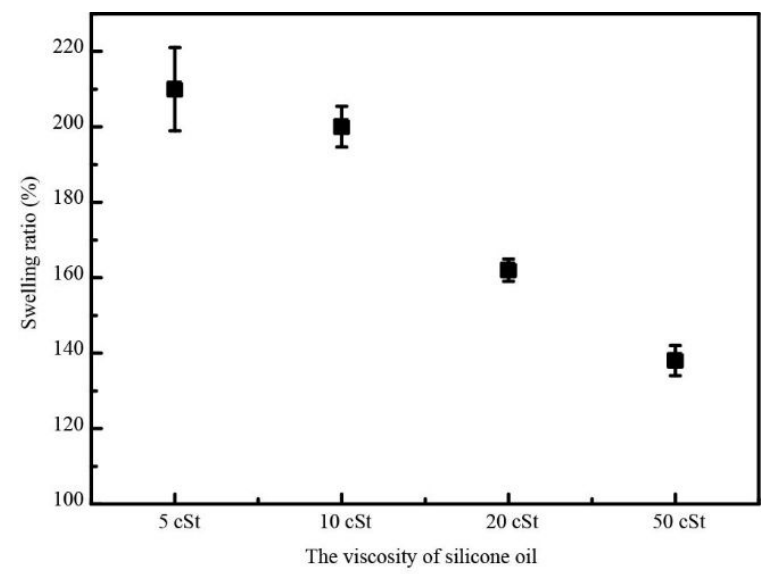

Figure S11. The swelling rate of uPDMS gels swelled with different viscosity silicone oil at $80{ }^{\circ} \mathrm{C}$ 


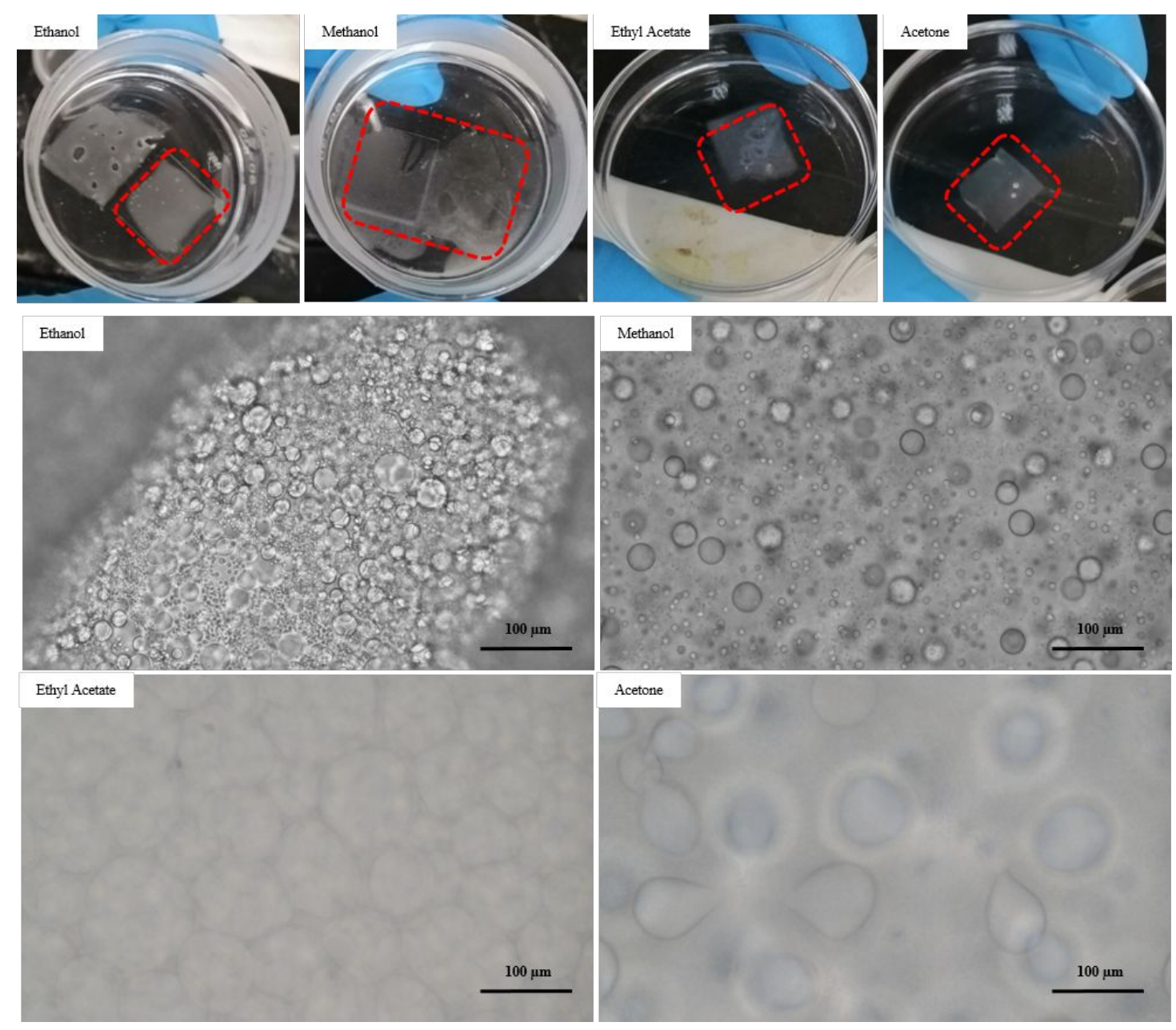

Figure S12. Optical microscopy image and the photograph of uPDMS films swelled in different solvents at room temperature. Some solvent (such ethanol, methanol, ethyl acetate, acetone) can form the droplets by swellon film 


\begin{tabular}{ccccc}
\hline & Mw & Mn & Mp & PDI \\
\hline PDMS-NH $\left(3000^{\mathrm{a}}\right)$ & $4.0 \times 10^{3}$ & $1.8 \times 10^{3}$ & $3.7 \times 10^{3}$ & 2.28 \\
uPDMS & $5.9 \times 10^{4}$ & $3.2 \times 10^{4}$ & $5.6 \times 10^{4}$ & 1.83 \\
\hline
\end{tabular}

${ }^{a}$ Molecular weight according to Gelest product specifications

Table S1. GPC results of uPDMS

\section{Supplementary References}

(1) Dokukin, M. E.; Sokolov, I. Quantitative Mapping of the Elastic Modulus of Soft Materials with HarmoniX and PeakForce QNM AFM Modes Langmuir 2012, 28, 16060-16071.

(2) Cui, J.; Daniel, D.; Grinthal, A.; Lin, K.; Aizenberg, J. Dynamic Polymer Systems with SelfRegulated Secretion for the Control of Surface Properties and Material Healing. Nat. Mater. 2015, 14, 790-795.

(3) Bai F.; Yang X.; Huang W. Synthesis of Narrow or Monodisperse Poly(divinylbenzene) Microspheres by Distillation-Precipitation Polymerization. Macromolecules 2004, 37, 9746-9752.

\section{Supplementary Video}

Video S1 To show the dynamic motion of a water droplet on the sample at different conditions. 